\title{
An examination of saltatory predation strategies employed by fish larvae foraging in a variety of different turbulent regimes
}

\author{
D. M. Lewis*, S. I. Bala \\ Department of Applied Mathematics, University of Liverpool, M \& O Building, Peach Street, Liverpool L69 7ZL, UK
}

\begin{abstract}
Saltatory or pause-travel predation strategies are exhibited by a wide variety of different species of fish larvae (e.g. Atlantic cod Gadus morhua and herring Clupea harengus, white crappie Pomoxis annularis and red drum Sciaenops ocellatus), usually classified as visual predators. Given the frequency that saltatory predation behaviour is observed, one would expect that this type of foraging provides an optimal balance between the energy expended whilst swimming, as against the potential energy to be gained upon successfully capturing some suitable prey. However, visual predators must live and forage in the upper 10s of metres of the ocean mixed layer, where light levels are relatively high, which means that their ability to find and capture prey is strongly influenced by small-scale turbulence. The strength of the turbulence regime characterising the water motion of the mixed layer is subject to considerable variation, but given the commonalty of saltatory predation, one would suspect that it remains a robust strategy under a variety of different conditions. This paper seeks to address this issue by studying the impact of saltatory predation over a wide cross section of turbulent environments. By means of a combination of kinematic simulations and theoretical predictions, the paper demonstrates that saltatory predation is indeed a robust and optimal strategy in the sense described, provided the larva's travel speed is not too high. However, when subject to very high levels of turbulence a larva's net energy gain peaks and then falls, leading to a prediction that it would either cease foraging altogether, or seek an escape from such an environment.
\end{abstract}

KEY WORDS: Larval foraging · Turbulence Restricted perception fields · Net energy gain Resale or republication not permitted without written consent of the publisher

\section{INTRODUCTION}

Experimental observations of larval fish performing saltatory or pause-travel search patterns have been recorded across a wide variety of different species (e.g. Rosenthal \& Hempel 1970, Sundby \& Fossum 1990, Browman \& O'Brien 1992a,b, Coughlin et al. 1992, MacKenzie \& Kiørboe 1995, von Herbing \& Gallager 2000, Galbraith et al. 2004, Lough et al. 2005). With such a wide range of observations, the question arises as to why such a foraging strategy is presumably more advantageous in terms of higher survival and maturation probability vis-à-vis alternatives, such as cruise (continuous swimming) or ambush feeding. Larval fish are visual predators (e.g. Porter et al. 2005, Peck et al.
2006), and consequently they must concentrate their foraging activity in the upper $10 \mathrm{~s}$ of metres of the ocean mixed layer, where irradiance levels are sufficiently high for them to detect their prey (Paul 1983). However, the upper mixed layer is turbulent and subject to considerable variations in wind or tidal forcing, which means that larval fish must continue to forage successfully in a dynamic fluid environment encapsulated by a wide range of statistical descriptors. Hence, given that a fish larva has relatively limited sensory capabilities for assessing and responding to changes in the characteristics of the turbulent flow, one would presume that pause-travelling must remain relatively robust and optimal in some sense when subjected to significant variations in the local fluid environment. 
Since Rothschild \& Osborn (1988) first raised the question concerning how small-scale turbulence might impact planktonic contact and capture rates, considerable research effort has been devoted to further understanding of the problem and to formulate predictive models. References pertaining specifically to larval fish include MacKenzie \& Leggett (1991), MacKenzie et al. (1994), Muelbert et al. (1994), Fiksen et al. (1998), Visser \& MacKenzie (1998), Leising \& Franks (1999) and MacKenzie \& Kiørboe (2000). More theoretical ideas pertaining to planktonic contact/capture rates in turbulent flows include the works of Yamazaki et al. (1991), Lewis \& Pedley (2000, 2001), Mann et al. (2002) and Metcalfe et al. (2004), although given the inherent difficulties associated with the problem, the resulting models tend to struggle to get beyond all-embracing generalizations to make specific biologically relevant predictions.

Most of the current planktonic encounter/capture rate models that incorporate turbulence are based on the assumption that an encounter (Gerritsen \& Strickler 1977) between a predator and its potential prey occurs when their relative velocity (made up of contributions from their individual swimming speeds and the flow field itself) carries them to within some fixed separation distance $R$ (usually between 1 and $40 \mathrm{~mm}$ ) of each other. This definition implies that the predator's perception capabilities are spherically symmetric, which somewhat simplifies the calculations required to make theoretical predictions. However, experimental observations of the predatory behaviour of copepods by Bundy et al. (1998), and fish larvae by Browman \& O'Brien (1992b), clearly indicate that they tend to perceive potential prey at much greater frequencies when their relative orientations lie within a small range of specific angles. Hence, planktonic perception capabilities are, in general, much more restricted than spherically symmetric case. Browman \& Skiftesvik (1996) and Galbraith et al. (2004) describe the typical perception field of an Atlantic cod Gadus morhua larva as resembling a forward-projecting wedge of $10 \mathrm{~mm}$ radius (horizontal half angle $45^{\circ}$, vertical half angle $10^{\circ}$ ) projecting above the body axis (see Fig. 1a). Such a drastic geometrical divergence from a spherical (or indeed hemispherical) perception field (representing as it does an approximate factor of 23 reduction in search volume over that of a sphere with similar radius), must have an impact on the type of strategy best suited for such a larval predator to adopt, in order to optimise its foraging success rate. Model predictions of foraging strategies based on predators with spherical perception fields cannot be expected to be applicable to fish larvae with such limited search volumes.

Theoretical work on planktonic predation in turbulent flows for predators with restricted perception

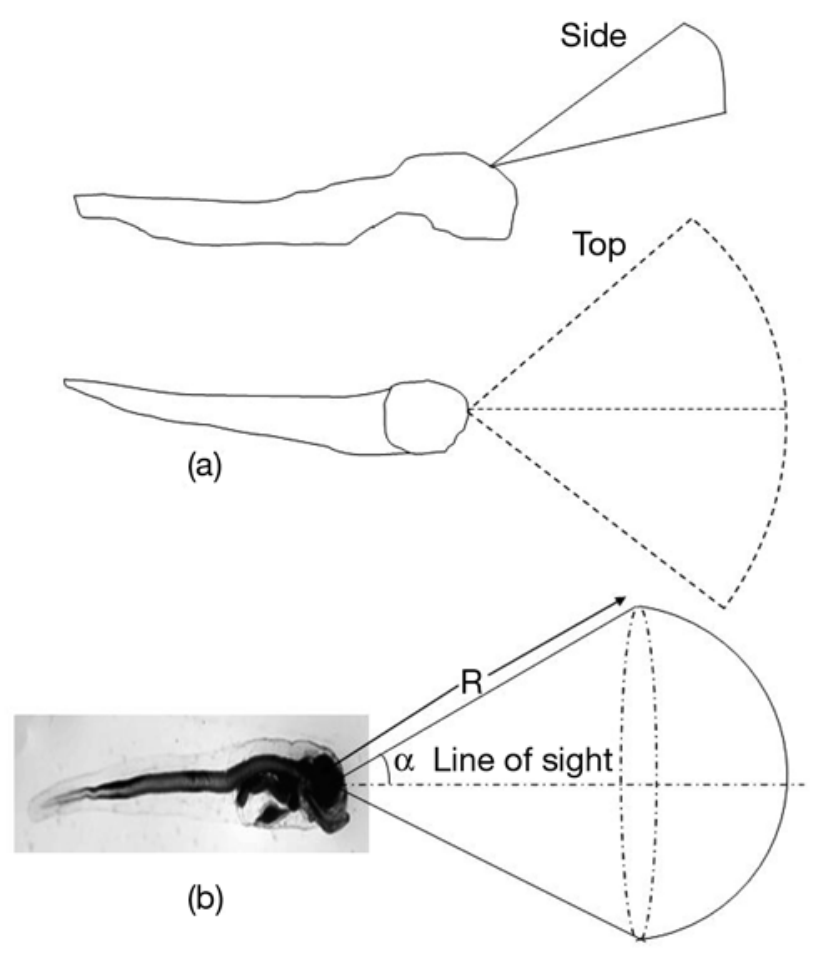

Fig. 1. Schematic diagram of approximate perception fields: (a) a cod Gadus morhua larva wedge and (b) the right circular cone of semi-vertex angle $\alpha$, combined with spherical cap of contact radius $R$, for the idealised predator used in the model

fields is considerably more difficult than for the case of spherical geometry, although there have been recent advances (e.g. Lewis 2003, Mann et al. 2006). In another recent paper, Lewis \& Bala (2006) investigated predation rates subject to turbulence for planktonic predators with restricted perception fields in the form of a cone, radius $R$, semi-vertex angle $\alpha$, directed along the predator's swimming direction (see Fig. 1b). Part of that study involved simulating the movements of an imaginary Atlantic cod larva following a pause-travel foraging strategy, and estimating its predation rate in an environment in which the prey density was set to $4.2 \mathrm{l}^{-1}$ (a low but not unrealistic level for the ocean mixed layer) and subject to turbulence. Observations of Galbraith et al. (2004) suggest that an Atlantic cod larva's pause-travel mode lasts about $3 \mathrm{~s}$, with $2 \mathrm{~s}$ spent swimming at a relatively high average speed of $5 \mathrm{~mm}$ $\mathrm{s}^{-1}\left(\sim 1 \mathrm{BL} \mathrm{s}^{-1}\right)$, compared to $1 \mathrm{~s}$ spent pausing and scanning for prey. Simulations were conducted that mimicked this behaviour, except that the $3 \mathrm{~s}$ period was split up into 4 different pause-travel ratios $2 / 3: 1 / 3,1 / 2: 1 / 2$, $1 / 3: 2 / 3$ and $1 / 6: 5 / 6$. Predation rates were estimated assuming that the larvae required an average handling time of $1 \mathrm{~s}$ to fixate, pursue and attack a potential prey meso-zooplankton that might be advected into its per- 
ception field. The resulting predation rates were utilised in a net energy gain (NEG) model of Galbraith et al. (2004) to estimate the difference between the energy expenditure of the larva during its pause-travel swimming, compared to the energy it would gain from successfully capturing its prey (specifically assumed to be Calanus finmarchicus nauplius). A plot (from Lewis $\&$ Bala 2006) of the resulting NEG against pause-travel ratios for a variety of perception angles is shown in Fig. 2. The figure highlights two key points. First for the predators with the widest perception fields $\alpha=\pi / 4$, $\pi / 3$, saltatory predation is not an attractive option. They can gain most energy simply by employing a cruising, 1:0, foraging strategy, in which they continually swim at their average speed of $5 \mathrm{~mm} \mathrm{~s}^{-1}$, changing their swimming heading every $3 \mathrm{~s}$ or so, until they encounter a potential prey. However, for the predator with the most restricted perception field $\alpha=\pi / 6$ (which corresponds most closely to the actual wedge-shaped perception field believed to pertain to the Atlantic cod

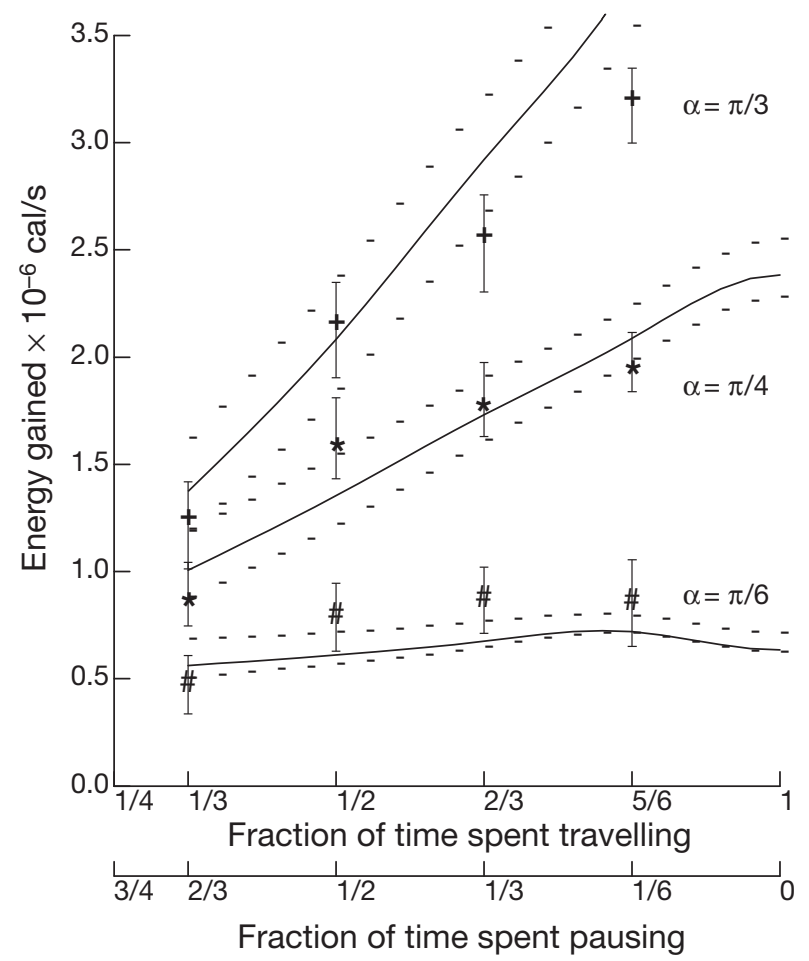

Fig. 2. Variation in the net energy gain against the pausetravel ratio for Atlantic cod Gadus morhua larvae with a variety of relatively narrow perception angles $\alpha$. Flow and fluid parameters: energy dissipation rate $\langle\varepsilon\rangle=5.53 \times 10^{-9} \mathrm{~m}^{2} \mathrm{~s}^{-3}$ and viscosity $v=1.0 \times 10^{-6} \mathrm{~m}^{2} \mathrm{~s}^{-1}$. An energy expenditure whilst swimming equivalent to $E_{S}=2.73 \times 10^{-6} \mathrm{cal} \mathrm{mg}^{-1} \mathrm{~mm}^{-1}$, is assumed. The points marked by plus signs $(\pi / 3)$, asterisks $(\pi / 4)$ and hashes $(\pi / 6)$, and their associated error bars are derived from the kinematic simulations. Solid lines: corresponding predicted energy gains; surrounding dashed lines: associated standard deviations for these predictions (see Fig. 7 in Lewis \& Bala 2006) larva), the model simulations and theoretical predictions suggest that the maximum energy gain can be attained following something close to a $1 / 3: 2 / 3$ pausetravel strategy. This is exactly the type of strategy employed by the cod larvae observed by Galbraith et al. (2004), although MacKenzie \& Kiørboe (1995) highlight observations of cod and herring Clupea harengus larvae pausing for longer periods (closer to 2/3:1/3) than this.

Fig. 2 presents quite a striking prediction. However, the results and simulations were based on calculations for just one specific turbulent flow field characterised by an average energy dissipation rate of $\langle\varepsilon\rangle=5.53 \times$ $10^{-9} \mathrm{~m}^{2} \mathrm{~s}^{-3}$ (characteristic of the lower levels of the mixed layer near the thermocline perhaps 15 to $100 \mathrm{~m}$ deep) and a kinematic viscosity of $\mathrm{v}=1.0 \times 10^{-6} \mathrm{~m}^{2} \mathrm{~s}^{-1}$ (corresponding to a sea temperature of about $20^{\circ} \mathrm{C}$ ). Many species of fish larva live in waters much colder and more viscous than this, leading to reductions in their metabolic rate (von Herbing \& Boutilier 1996) and to a corresponding degradation in their ability to sustain bouts of relatively fast swimming for long periods (Fuiman \& Batty 1997, von Herbing 2002). The natural variability of their environment means that fish larvae must be exposed to turbulent conditions characterised by higher average values of $\langle\varepsilon\rangle$. Consequently, if the prediction highlighted in Fig. 2 is to have real merit, pause-travel foraging strategies should remain energetically favourable across a range of different dynamical regimes.

The aim of the present paper is to examine this point by simulating a variety of pause-travel modes across a range of turbulent regimes by varying both the viscosity and energy dissipation rate. The various turbulent regimes will be modelled using kinematic simulations, each containing a variety of predator and prey 'particles', which are moved around by means of a combination of swimming and advection by the flow. Certain characteristics of the fish larva are fixed; primarily its perception field angle will be restricted to the narrow value of $\alpha=\pi / 6$, with constant contact radius, which is closest in volume (a factor of 15 reduction compared to the corresponding sphere) to what appears to be the actual wedge-shaped perception field. As Fig. 2 illustrates that saltatory predation for larger $\alpha$ values appears energetically unfavourable, this is not an unreasonable assumption. However, the travel swimming speed will be varied, with greater emphasis placed on the lower values found in colder and more viscous conditions. Along with the results of kinematic simulations, theoretical predation rate predictions will appear, derived from modelling ideas fully discussed in Lewis (2003) and Lewis \& Bala (2006). The results of the predation rate calculations, both simulations and predictions, are then substituted into the NEG model 
in order to examine the robustness of pause-travel as a foraging strategy, and what trends might be observed in the ratio of the pausing to travelling modes for the strategy to remain optimal. This depends critically on the level of energy expended during the travel phase, and calculations are undertaken to estimate the influence of viscosity and energy dissipation rate on this term, and how these parameters may manifest themselves in changes to a larva's foraging behaviour.

\section{KINEMATIC SIMULATIONS}

Kinematic simulations have become a widely used tool for studying the influence of turbulence on the behaviour of micro/meso-zooplankton and fish larva, providing a good representation of a homogeneous-isotropic turbulent flow (a good approximation at planktonic contact scales), without the extra computational expense of solving the Navier-Stokes equations directly. Along with the models by Lewis \& Pedley $(2000,2001)$ developed to examine the encounter/predation rates mentioned earlier, kinematic simulations have also been adapted to examine the dispersion of chemical trails laid by copepods (see Visser \& Jackson 2004).

The kinematic simulations carried out here are identical in formulation to those discussed in Lewis \& Bala (2006). Briefly, the representative turbulent velocity field is constructed by a superposition of $N_{K}$ Fourier modes:

$$
\begin{aligned}
& \boldsymbol{W}(\boldsymbol{x}, t)= \\
& \sum_{n=1}^{N_{k}}\left[\left(\boldsymbol{a}_{n} \times \hat{\boldsymbol{k}}_{n}\right) \cos \left(k_{n} \cdot \boldsymbol{x}+\omega_{n} t\right)+\left(\boldsymbol{b}_{n} \times \hat{\boldsymbol{k}}_{n}\right) \sin \left(\boldsymbol{k}_{n} \cdot \boldsymbol{x}+\omega_{n} t\right)\right]
\end{aligned}
$$

(see Fung et al. 1992, Malik \& Vassilicos 1999). Here, $\boldsymbol{k}_{n}=\boldsymbol{k}_{n} \hat{\boldsymbol{k}}_{n}$ represent wave vectors (essentially a measure of the reciprocal of the length scale of the eddies, or swirling motions, constituting the flow) and $\omega_{n}$ represents frequency modes (a measure of the reciprocal of the time scale for an eddy to complete a revolution), both of which depend upon the form of the turbulent kinetic energy distribution $E(k)$. This is a measure of how much energy is contained in an eddy of size $1 / \mathrm{k}$. Large eddies tend to be slowly revolving, but energy rich, generated as they are by the driving forces of the flow. Small eddies revolve quickly, but are energy depleted, as at small scales energy is dissipated into heat by viscosity (Tennekes \& Lumley 1972). By judicious choice of the random vectors $\boldsymbol{a}_{n}$ and $\boldsymbol{b}_{n}$ one can construct a 'synthetic' flow field with many of the kinematic properties of a real velocity field satisfying the full Navier-Stokes equations. Hence, for a homogeneous and isotropic turbulent flow of interest here, the directions of the unit wave vectors $\hat{\boldsymbol{k}}_{n}$ map out the surface of a unit sphere, whilst the orientations of $\boldsymbol{a}_{n}$ and $\boldsymbol{b}_{n}$ are chosen to be normal to $\hat{\boldsymbol{k}}_{n}$, so guaranteeing incompressibility. The magnitudes of the vectors $\boldsymbol{a}_{n}$ and $\boldsymbol{b}_{n}$ are equal, and must satisfy:

$$
\sum_{n=1}^{N_{k}}\left|\boldsymbol{a}_{n}\right|^{2}=\sum_{n=1}^{N_{k}}\left|\boldsymbol{b}_{n}\right|^{2}=2 \int_{0}^{\infty} E(k) \mathrm{d} k
$$

where $E(k)$ is a given turbulent kinetic energy spectrum. The frequency modes satisfy $\omega_{n}=0.4 \sqrt{k_{n}^{3} E\left(k_{n}\right)}$. As predators and prey can potentially move very close to one another, it is desirable that $E(k)$ gives a good representation of the energy distribution of the flow at high wave numbers. To this end, the energy spectrum model of Mei \& Adrian (1995), viz.

$$
E(k)=\frac{1.5 C W^{2} k^{4} L^{5} \mathrm{e}^{-k^{2} \eta^{2}}}{\left[1+(k L)^{2}\right]^{17 / 6}}, \quad k \in\left[\frac{1}{4 L}, \frac{2}{\eta}\right]
$$

where $C$ is a normalisation constant and $L$ is a representative length scale of the largest eddies, was substituted. This spectrum behaves like $k^{4}$ for $k \leq(1 / L)$ (Batchelor 1953), like $k^{-5 / 3}$ in the inertial subrange and dies off exponentially at large $k$ when the flow is dominated by viscosity. Eq. (3) gives a very good representation of the general characteristics of $E(k)$ observed in experiments.

For this study a total of 6 separate flow fields (see Table 1) were constructed from Eqs. (1) to (3), charac-

\begin{tabular}{|c|c|c|c|c|c|c|c|c|c|}
\hline Flow & $\begin{array}{c}\langle\varepsilon\rangle \\
\left(\mathrm{m}^{2} \mathrm{~s}^{-3}\right)\end{array}$ & $\begin{array}{c}v \\
\left(m^{2} s^{-1}\right)\end{array}$ & $\begin{array}{c}\eta=\left(v^{3} /\langle\varepsilon\rangle\right)^{1 / 4} \\
(\mathrm{~m})\end{array}$ & $\begin{array}{c}v_{K}=(v /\langle\varepsilon\rangle)^{1 / 4} \\
\left(\mathrm{~m} \mathrm{~s}^{-1}\right)\end{array}$ & $\begin{array}{c}\tau_{K}=(\nu /\langle\varepsilon\rangle)^{1 / 2} \\
(\mathrm{~s})\end{array}$ & $W$ & $L$ & $\lambda$ & $\operatorname{Re}_{\lambda}$ \\
\hline 1 & $5.53 \times 10^{-9}$ & $1.0 \times 10^{-6}$ & $3.67 \times 10^{-3}$ & $2.72 \times 10^{-4}$ & 13.45 & 2.73 & 20.3 & 10.56 & 28.8 \\
\hline 2 & $5.53 \times 10^{-9}$ & $1.5 \times 10^{-6}$ & $4.97 \times 10^{-3}$ & $3.02 \times 10^{-4}$ & 16.47 & 2.46 & 15.0 & 9.54 & 23.5 \\
\hline 3 & $5.53 \times 10^{-8}$ & $1.0 \times 10^{-6}$ & $2.06 \times 10^{-3}$ & $4.85 \times 10^{-4}$ & 4.25 & 4.85 & 114.0 & 18.78 & 91.1 \\
\hline 4 & $5.53 \times 10^{-8}$ & $1.5 \times 10^{-6}$ & $2.80 \times 10^{-3}$ & $5.37 \times 10^{-4}$ & 5.21 & 4.38 & 84.1 & 16.97 & 74.4 \\
\hline 5 & $5.53 \times 10^{-7}$ & $1.0 \times 10^{-6}$ & $1.16 \times 10^{-3}$ & $8.62 \times 10^{-4}$ & 1.34 & 8.62 & 641.3 & 33.40 & 288.0 \\
\hline 6 & $5.53 \times 10^{-7}$ & $1.5 \times 10^{-6}$ & $1.57 \times 10^{-3}$ & $9.54 \times 10^{-4}$ & 1.65 & 7.79 & 473.1 & 30.18 & 235.2 \\
\hline
\end{tabular}

Table 1. Kolmogorov length, velocity and time scales, and parameter values characterising the 6 kinematic flow fields used in the simulations. $W$ is the Eulerian velocity scale, $L$ is the large eddy scale, $\lambda=\left(15 v W^{2} /\langle\varepsilon\rangle\right)^{1 / 2}$ the Taylor micro-scale and $\operatorname{Re}_{\lambda}=W \lambda / v$ the Taylor Reynolds number (values are in non-dimensional units except where stated) 
terised by 3 energy dissipation rates $\langle\varepsilon\rangle=5.53 \times 10^{-9}$, $5.53 \times 10^{-8}$ and $5.53 \times 10^{-7} \mathrm{~m}^{2} \mathrm{~s}^{-1}$, and 2 viscosity $v$ values, $1.0 \times 10^{-6}$ and $1.5 \times 10^{-6} \mathrm{~m}^{2} \mathrm{~s}^{-1}$. The former represent increasingly energetic flow regimes characteristic of the upper mixed layer a few metres below the surface (although not as violent as might be experienced in coastal zones, or the uppermost few metres of the water column when subject to very strong wind forcing $>10 \mathrm{~m} \mathrm{~s}^{-1}$, for reasons to be discussed), whilst the latter represent variations in sea surface temperature from about 20 to $5^{\circ} \mathrm{C}$, respectively. This variation in viscosity/temperature values reflects the aim to investigate the general robustness of pause-travel as a successful foraging strategy. One would not expect one individual fish larva to experience such a diverse range of temperature regimes during its larval phase, and consequently the exact details of the particular saltatory strategy it might pursue may be adapted to suit a smaller range of values.

Using these parameter values for $\langle\varepsilon\rangle$ and $v$, one can calculate the Kolmogorov scales for length, velocity and time (defined and listed in Table 1) for each flow, which will be used to non-dimensionalize all the remaining parameters and calculations. Into each flow, 2 types of planktonic 'particles' are introduced, one to represent predatory fish larvae and the other its prey. The terminology 'particle' in this context means that the length scales associated with both larvae and prey are smaller than the various Kolmogorov length scales $\eta=\left(v^{3} / \varepsilon\right)^{1 / 4}$, with the consequence that their presence will have negligible impact on the general characteristics of the flow field in question. In this work an Atlantic cod Gadus morhua larva of length $5 \mathrm{~mm}$ (roughly 10 to $20 \mathrm{~d}$ post-hatching) will serve as the predatory template for the various foraging parameters. A larval length of $5 \mathrm{~mm}$ is somewhat larger than the typical Kolmogorov length scale (see Table 1), so the assumption that larvae can be modelled essentially as point particles is somewhat erroneous. However, the number of larvae incorporated into each flow (512 simulation $^{-1}$ ) is at such low densities that one would not expect their presence to materially alter the characteristics of the flow to the extent that the simulated number of prey encounters would differ from those in a real ocean mixed layer. Prey captures would be influenced by distortions in the local flow field brought about by the relatively large size of the larvae, but, in this work, capture rates are estimated by statistical means, which avoids the necessity of performing detailed computations of the flow field around the larva's body as it moves to intercept its prey. The nauplii of the copepod Calanus finmarchicus serve as a template for the planktonic prey 'particles'. They are considerably $<1 \mathrm{~mm}$ in size, extremely abundant in the North Atlantic, and form an important part of the diet of both $\operatorname{cod}$ G. morhua and herring Clupea harengus larvae.

Certain key parameters were kept constant throughout the various simulations, and the principal values are listed in Table 2. First, the perception angle $\alpha$ was fixed at $\pi / 6$ for the reasons discussed earlier. Second, the contact radius was fixed at $12 \mathrm{~mm}$ in line with previous work (Galbraith et al. 2004 suggest a figure of $10 \mathrm{~mm}$, although this is an average and will probably depend on the age of the larvae). Prey density was also fixed at a value of $4.3 \mathrm{l}^{-1}$, characteristic of the pelagic marine environment, although it is a relatively low figure compared with many experimental observations (e.g. Browman \& O'Brien 1992b). A single simulation consisted of 10 runs, starting from different random sets of starting positions and swimming velocities of the various planktonic 'particles', from which mean and variance statistics were calculated. Each set of simulations was carried out 4 times, over increasingly large cubic domains containing increasing numbers from 512 to 16384 of prey. (The cube dimension 1 was adjusted to ensure the prey density remains constant, with the smallest value of $l$ chosen to ensure it exceeds $L$ listed in Table 1.) The averages can then be extrapolated to estimate the results for a cube of infinite dimension. This should negate the influence of the boundary conditions employed; predators and prey that swim out of the simulation domain must be recreated elsewhere, which tends to bias the encounter rate upwards (see Lewis \& Pedley 2000). Finally, the total duration of a single pause-travel step (that is the combined time of 1 travelling motion plus 1 pause phase) performed by the larva was fixed at $3 \mathrm{~s}$, as observed by Galbraith et al. (2004).

The details of the swimming and pausing motions characterising the make-up of a specific saltatory for-

Table 2. Values (in non-dimensional units) for certain parameters needed to make the theoretical predictions shown in figures. The flow numbers refer to the 6 flow fields in Table 1

\begin{tabular}{|lrrrrr|}
\hline Flow & $\tau_{L}$ (Eq. 6) & $R \equiv 12 \mathrm{~mm}$ & $\tau_{\text {dep }}(R)^{\mathrm{a}}$ & $W_{T}^{\mathrm{b}}$ & $T_{R} \equiv 1 \mathrm{~s}$ \\
\hline 1 & $1.92 \pm 0.43$ & 3.20 & 2.30 & 1.43 & 0.074 \\
2 & $1.49 \pm 0.68$ & 2.40 & 2.83 & 1.15 & 0.061 \\
3 & $3.09 \pm 1.16$ & 5.82 & 2.57 & 2.03 & 0.235 \\
4 & $2.80 \pm 1.09$ & 4.29 & 2.22 & 1.69 & 0.192 \\
5 & $4.40 \pm 1.10$ & 10.35 & 3.18 & 2.73 & 0.746 \\
6 & $4.20 \pm 1.12$ & 7.63 & 2.47 & 2.33 & 0.606 \\
\multicolumn{2}{l}{ a Defined by Lewis (2003, his Eq. 16) } & & & \\
befined by Lewis (2003, his Eq. 17) & & & \\
\hline
\end{tabular}


aging strategy were varied across the different flow fields. Firstly, the ratio of time spent pausing to travelling during a single $3 \mathrm{~s}$ pause-travel step was varied in 4 ways from (2/3 pausing, $1 / 3$ travelling), $(1 / 2,1 / 2),(1 / 3,2 / 3)$ and $(1 / 6,5 / 6)$. Secondly, the average swimming speed of the larval predators during the travel phase was varied from 3,5 up to $10 \mathrm{~mm} \mathrm{~s}^{-1}$, or 0.6 to $2.0 \mathrm{BL} \mathrm{s}^{-1}$. (The actual swimming velocity of an individual larva is drawn from 3-dimensional, isotropic, Gaussian distributions with zero means and standard deviations $\sigma_{P}$, chosen so that the average swimming speed $\left\langle v_{P}\right\rangle=\sqrt{8 / \pi} \sigma_{P}$ corresponds to the values above.) On reaching the end of a travel phase, the larva's heading was changed and its swimming speed restricted to a very low 'pause' value $\left(\sim 0.1 \mathrm{~mm} \mathrm{~s}^{-1}\right)$. Observations by von Herbing \& Gallager (2000) and von Herbing et al. (2001) show that some 'pauses' are phases where a larva performs a slow speed ( 0.2 BL $\mathrm{s}^{-1}$ ) glide, although the detailed behaviour depends on the larval size. So allowing some swimming motion during pauses was designed to take this behaviour into account. At the end of the pause phase, the larva's heading was changed again as it accelerates back up to its travel speed (drawn from the appropriate distribution as before). Planktonic 'particle' trajectories $\boldsymbol{X}_{P / H}(t)$ ( $P$ denotes predator, $H$ denotes prey) were obtained by integrating:

$$
\frac{\mathrm{d} \boldsymbol{X}_{P / H}}{\mathrm{~d} t}=\boldsymbol{w}\left[\boldsymbol{X}_{P / H}, t\right]+\boldsymbol{v}_{P / H}(t)
$$

using a fourth order Runge-Kutta method, where $\boldsymbol{w}(\boldsymbol{x}, t)$ is the turbulent flow field at position $\boldsymbol{x}$ and time $t$, and $\boldsymbol{v}_{P / H}(t)$ represents the current swimming velocities of the predator (pausing or travelling) and prey, respectively ( $v_{H}=0$ throughout).

Every time a prey micro-organism moved within the perception field of a predator, a 'contact' was recorded. It was assumed that each of these contacts would act as a trigger for a pursuit and attack response on the part of the larva. Munk (1995), MacKenzie \& Kiørboe (2000) and von Herbing et al. (2001) describe just such behaviour in cod larvae. Principally, it consists of the larva changing direction to pursue the prey, sometimes altering its relative orientation better to fixate the prey in its field of view, before opening its mouth and sucking the prey towards it. Attacks occur when the predator and prey are in very close proximity, $\leq 2 \mathrm{~mm}$, and are usually successful (MacKenzie \& Kiørboe 2000 report a $91 \%$ success rate), irrespective of turbulence conditions (at these distances the component of the fluid flow making up the relative velocity between predator and prey will be small, and dominated by viscous rather than inertial terms). Pursuits tend to be much less suc- cessful, as the prey will frequently be advected away from the larva by the flow in such a way that it cannot move close enough to initiate an attack. Pursuit and attack success rates combine to give a capture probability for an encountered prey. There are a variety of estimates reported in the literature (30 to $40 \%$ by MacKenzie \& Kiørboe 1995, 70\% by MacKenzie \& Kiørboe 2000, $50 \%$ by von Herbing et al. 2001) depending on larval size, prey escape responses and flow conditions. To attempt to model in detail the pursuit and attack responses of a larva encountering a prey, in combination with associated flow fields that result, is too computationally expensive an undertaking to be contemplated here. So a more pragmatic approach is adopted, and the results of the various pursuit and attack responses are encapsulated into a single probability of capture function $p_{C A P}$. As in Lewis \& Bala (2006) this will be defined as:

$$
p_{C A P}=\frac{t_{T R A}^{2}}{t_{T R A}^{2}+T_{R}^{2}}
$$

Here, $t_{T R A}$ is the time the prey spends inside the larva's perception field assuming the latter initiates no pursuit response, and $T_{R}$ is a reaction time scale, which encapsulates the average length of time the larva needs to complete a pursuit and attack for a successful capture. If $t_{T R A} \gg T_{R}$, capture is highly probable; if $t_{T R A} \ll T_{R}$ the prey will almost certainly escape. Von Herbing \& Gallager (2000) record estimates of pursuit and attack responses lasting for a total of about $1 \mathrm{~s}$, although the observations of MacKenzie \& Kiørboe (2000) show that pursuits may continue for longer, between 2 and $4 \mathrm{~s}$. There is no unconditional way of translating these values into a prediction for $T_{R}$, but, given average pursuit speeds of between 5.6 and $9.4 \mathrm{~mm} \mathrm{~s}^{-1}$ (von Herbing et al. 2001) and a maximum initial perception distance $R=12 \mathrm{~mm}$, it is clear that any prey lingering in the larva's perception field for more than about $1 \mathrm{~s}$ stands a good chance of being captured, and so this value was adopted here. During the simulations, once an initial contact was recorded a check on the value of $t_{T R A}$ was made, until the prey moved out of the larva's perception field. At that point, a simple calculation of the likely capture probability was made from (Eq. 5), with $T_{R}=1 \mathrm{~s}$. By comparing this result with a random uniform deviate drawn from the range [0,1], it is then possible to make a definitive prediction as to whether the prey would have been captured. If so, the prey was removed from the simulation, but a new one was spontaneously recreated in a different part of the simulation domain (away from any larvae), to ensure the overall prey density remained fixed. If the prey was found to have escaped, it was allowed to continue moving as before. 


\section{THEORETICAL PREDICTIONS}

The adoption of a conical shape to describe the larva's perception field, in conjunction with the use of a capture function (Eq. 5) to describe the outcome of its pursuit and attack responses, means that the results of the simulations are amenable to theoretical prediction. Two key modelling concepts form the basis of the predictions, and were the subject of 2 earlier papers (Lewis 2003 [for encounter rates] and Lewis \& Bala 2006 [for predation rates]). First, an exact geometrical prescription of the volume of water encompassed by the predator's perception field can be constructed assuming the predator follows (through a combination of swimming and advection by the flow) a straight line trajectory. This is important as the volume of fluid encompassed by the predator is directly related to the number of prey it encounters via the prey density. Second, computer predictions are made of how this volume changes when a predator follows an irregular path, which incorporate details of how often the predator changes its swimming heading and how the strength of the flow will impinge on its ability to control its direction of motion. The details are quite complex and mathematical, and will not be discussed in any depth here. Suffice it to say that the parameterisation and methodology described in the papers above, which looked at simulations conducted in one specific flow field, carry over to the different ranges of turbulent regimes discussed here. The only significant change is that the Langrangian integral time scale $\tau_{L}$ is used in place of the Langrangian 'contact' time scale $\tau_{L C}(R)$ (as defined in Lewis \& Pedley 2000, their Eqs. 54 to 56$)$. The former is defined by:

$$
\begin{aligned}
& \tau_{L}= \\
& \frac{1}{2} \int_{0}^{t}\left(1-\frac{T}{t}\right) \frac{\langle\boldsymbol{W}(\boldsymbol{X}(\boldsymbol{a}, t), t) \cdot \boldsymbol{W}(\boldsymbol{X}(\boldsymbol{a}, t+T), t+T)\rangle}{\left\langle|\boldsymbol{W}(\boldsymbol{X}(\boldsymbol{a}, t), t)|^{2}\right\rangle} \mathrm{d} T
\end{aligned}
$$

(Tennekes \& Lumley 1972), where $\boldsymbol{X}(\boldsymbol{a}, t)$ is the track of a fluid particle (found by integrating Eq. 4, with no swimming contribution) originating at position $\boldsymbol{X}=\boldsymbol{a}$ at $t=0$. This time scale depends purely on the flow field in question and helps to determine the effective interval time scale $\tau$ (see Lewis \& Bala 2006, their Eq. 23), an estimate of how much time must elapse for a predator to change, or 'forget', its previous heading. Substituting $\tau_{L}$ for $\tau_{L C}(R)$ gave more reliable predictions across various different flow fields (in previous work $\tau_{L C}(R)=$ $2.1 \pm 1.0$ was very similar to $\tau_{L}=1.92 \pm 0.43$ making little difference to the predictions). Table 2 lists values of $\tau_{L}$ and the various other parameters needed for the predictions, together with references to their definitions in earlier papers.

\section{RESULTS OF SIMULATIONS AND PREDICTIONS}

\section{Influence of travelling speed}

Fig. 3a shows the number of captures made by 3 different sets of 512 saltatory predators, swimming at 3 different average speeds, viz. 3, 5, and $10 \mathrm{~mm} \mathrm{~s}^{-1}(\sim 0.6$, 1 and $2 \mathrm{BL} \mathrm{s}^{-1}$ for a $5 \mathrm{~mm}$ larva) during their travel phase. The results are for a flow field with the same dissipation rate as in Fig. 2, but with a higher value of $v=1.5 \times 10^{-6} \mathrm{~m}^{2} \mathrm{~s}^{-1}$ (characteristic of a colder temperature of about $5^{\circ} \mathrm{C}$ ). Next to each plus sign, asterisk and hash mark indicating an average simulation result, a figure representing the percentage capture rate for the predators in their various pause-travel modes can be seen. (e.g. the number 43 in the bottom left hand corner is derived from the fact that the predators that

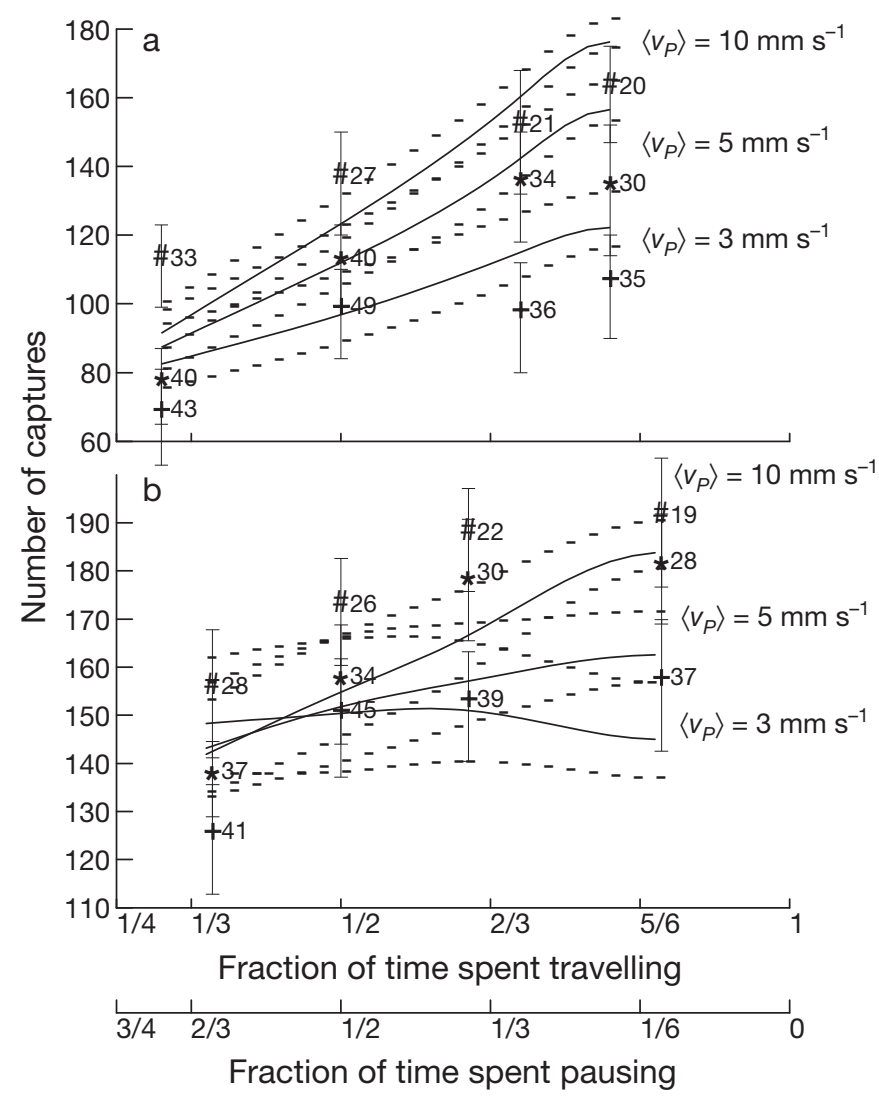

Fig. 3. Variation of the number of prey captures against the pause-travel ratio for $N_{P}=512$ saltatory predators, with average travel speeds $\left\langle v_{P}\right\rangle$ of 3,5 and $10 \mathrm{~mm} \mathrm{~s}^{-1}$. Viscosity $v=1.5 \times$ $10^{-6} \mathrm{~m}^{2} \mathrm{~s}^{-1}$, and energy dissipation rates: $(\mathrm{a})\langle\varepsilon\rangle=5.53 \times 10^{-9}$ $\mathrm{m}^{2} \mathrm{~s}^{-3}$ and $(\mathrm{b})\langle\varepsilon\rangle=5.53 \times 10^{-8} \mathrm{~m}^{2} \mathrm{~s}^{-3}$. Plus sign: travel speed of $3 \mathrm{~mm} \mathrm{~s}^{-1}$ : asterisk: $5 \mathrm{~mm} \mathrm{~s}^{-1}$; hash mark: $10 \mathrm{~mm} \mathrm{~s}^{-1}$ (These points and their associated error bars are derived from the kinematic simulations); small numbers close to the signs: capture efficiency as a percentage (see 'Influence of travelling speed'); solid lines: corresponding predicted mean number of captures; surrounding dashed lines: associated standard deviations for these predictions 
paused $70 \%$ of the time and travelled $30 \%$, at an average speed of $3 \mathrm{~mm} \mathrm{~s}^{-1}$, encountered an average of 157 prey and captured 67 of them, $\Rightarrow 67 \times 100 / 157 \approx 43 \%$ capture success). The solid line shows the corresponding theoretical predictions.

The most obvious point to note is that the larvae with the highest travelling speed capture most prey. This is simply because they encounter far more prey than the other predators (roughly 2.5 times as much as the larvae travelling at $3 \mathrm{~mm} \mathrm{~s}^{-1}$, and twice as much as those travelling at $5 \mathrm{~mm} \mathrm{~s}^{-1}$ ). Their capture efficiencies are reduced when compared to the slow swimmers (their velocity relative to the prey is larger, making capture more difficult), but because their reaction time scale $T_{R}$ is relatively fast, this reduction in their capture efficiency is modest, and not enough to totally offset the increased number of encounters they make. The overall capture rates of between 20 and $50 \%$ compare favourably with reported experimental values (specifically for larval cod preying on copepod nauplii) of 50 to $70 \%$ in still fluid (when captures are easier to make) from von Herbing et al. (2001), and of $22 \%$ from Munk (1995) under turbulent conditions. Hence, the estimated value of $T_{R}=1 \mathrm{~s}$ seems quite reasonable. Predation rates decline as pause duration increases, again because the number of encounters is reduced (roughly by a factor of 2 from the 1/6:5/6 mode to the $2 / 3: 1 / 3$ mode), although capture success increases as predator and prey will spend longer in close proximity during pauses. These trends are reflected in the model predictions, although there is a tendency to overestimate the number of captures for the $3 \mathrm{~mm} \mathrm{~s}^{-1}$ travellers, and underestimate the number for the $10 \mathrm{~mm} \mathrm{~s}^{-1}$ travellers.

Fig. $3 \mathrm{~b}$ shows the results of a similar set of simulations, but this time with the energy dissipation rate increased by a factor of 10 to $\langle\varepsilon\rangle=5.53 \times 10^{-8} \mathrm{~m}^{2} \mathrm{~s}^{-3}$. Generally, the more energetic flow brings about more encounters, leading to an increase in the number of captures made when compared to Fig. 3a. However, the increase is much more pronounced for the predation strategies dominated by pauses. This is because, if a larva is spending a higher proportion of the time pausing, its primary means of making encounters is through advection, and, consequently, its encounter/ capture rate depends critically on the characteristics of the flow. By contrast the encounters made by a larva following a 1/6:5/6 strategy, with a travel speed of $10 \mathrm{~mm} \mathrm{~s}^{-1}$, are more the result of swimming and less dependent on the flow. Encounters increase by only $26 \%$ in this instance, compared to $65 \%$ for the same larva following a 2/3:1/3 strategy.

Interestingly, capture success falls off only slightly in the more energetic flow field, and again with those predators pursuing long pause strategies being most sensitive to the change in $\langle\varepsilon\rangle$. This is somewhat surpris- ing, in that, it is usually assumed that increased levels of turbulence will make it significantly more difficult for a predator to capture an encountered prey. The explanation lies in the nature of the encounters themselves, and the geometry of the larva's perception field. For a conical perception field, as illustrated in Fig. 1b, encounters are possible both when a prey moves across the spherical base of the cone (when the initial contact distance is $R$ ), and if it moves into the cone from the sides (when the initial contact distance is $r<R$ ). The latter were termed 'close encounters' in Lewis (2003), a publication that includes mathematical expressions for the frequency of such encounters assuming the larva follows a straight line trajectory. Using these results it is possible to estimate the proportion of 'close encounters' that take place for different values of $\langle\varepsilon\rangle$. If the perception field is narrow as assumed here $(\alpha=\pi / 6)$, increasing $\langle\varepsilon\rangle$ significantly increases the number of close encounters. For instance, if a larva follows a 2/3:1/3 strategy and swims at $3 \mathrm{~mm} \mathrm{~s}^{-1}$, the contribution to the capture rate arising from 'close encounters' increases from about $2.5 \%$ at $\langle\varepsilon\rangle 5.53 \times 10^{-9} \mathrm{~m}^{2} \mathrm{~s}^{-3}$ to about $25 \%$ at $\langle\varepsilon\rangle 5.53 \times 10^{-8} \mathrm{~m}^{2}$ $\mathrm{s}^{-3}$ to about $40 \%$ at $\langle\varepsilon\rangle 5.53 \times 10^{-7} \mathrm{~m}^{2} \mathrm{~s}^{-3}$. Now the larval predators are no longer following straight line trajectories, so these figures should only be treated as rough estimates (changes in direction tend to increase the proportion of close encounters still further, see Fig. 6 in Lewis 2003), but the trend is clear. By their very nature, 'close encounters' are likely to be easier for the larva to capture, and it is this rise in the proportion of the former with $\langle\varepsilon\rangle$ that tends to offset the extra difficulty of making a capture attempt under more turbulent conditions. Hence, the relatively small overall decline in capture efficiency illustrated in Fig. 3b.

The question now arises as to how the associated predation rates derived from these figures translate into energy gains/losses for the fish larvae. The NEG model employed by Galbraith et al. (2004) was adapted from modelling ideas presented by Pitchford et al. (2003). Mathematically it can be expressed in the form:

$$
E=E_{C} P R-\left(E_{B}+E_{S}\left\langle v_{P}\right\rangle\right) M
$$

where $E$ is the net energy gained per unit time $\left(\mathrm{cal} \mathrm{s}^{-1}\right.$, where 1 calorie is equivalent to $4.18 \mathrm{~J}$ ). This is made up of 2 contributions. The positive energy gained from a prey capture is summarised by the $E_{C} P R$ term, where $E_{C}$ is the energy gained per capture $\left(1.97 \times 10^{-3} \mathrm{cal}\right.$, assuming larval prey consists principally of Calanus finmarchicus nauplii) and $P R$ is the predation rate. The associated energy expenditure required to sustain such a predation rate is summarised by the second term, which consists of a basal energy requirement 
$E_{B}=1.08 \times 10^{-6}$ (Galbraith et al. 2004) for a typical larva of mass $M=0.06 \mathrm{mg}$, and a cost of swimming $E_{S}\left\langle v_{P}\right\rangle$.

This term is a subject of considerable uncertainty. The value of $E_{S}=2.73 \times 10^{-6} \mathrm{cal} \mathrm{mg}^{-1} \mathrm{~mm}^{-1}$ put forward by Galbraith et al. (2004) is based on the idea that a larva expends most of its energy when it accelerates out of the low Reynolds number 'pause' phase into the relatively high Reynolds number 'travel' phase. They estimate that this requires an order of magnitude increase in energy expenditure in comparison with that required to swim at a constant speed in either Reynolds number regime (Fuiman \& Batty 1997). It may also help to explain the observations of von Herbing \& Gallager (2000) that cod larvae actually employ low-speed glides in the pause phase, rather than stopping altogether. The swimming cost must also be influenced by changes in the viscosity of the fluid and the strength of the flow field, although the exact nature of the dependence of $E_{S}$ on $v$ and $\langle\varepsilon\rangle$ is subject to much debate. Experiments conducted by Fuiman \& Batty (1997) on herring larvae show that average swimming speeds declined linearly from 15 to $5 \mathrm{~mm} \mathrm{~s}^{-1}$ as the viscosity was increased from $1.2 \times 10^{-6} \mathrm{~m}^{2} \mathrm{~s}^{-1}$ to $2 \times 10^{-6} \mathrm{~m}^{2} \mathrm{~s}^{-1}$. Wieser \& Kaufmann (1998) put forward the hypothesis, based on experiments on Danube bleak larvae, that this was due less to the influence of increased hydro-mechanical drag forces, as much as the muscle fibres in the larvae are insufficiently well developed to operate effectively in low temperatures. Their ideas are discussed further in von Herbing (2002). From these results, it is clear that high-viscosity values associated with relatively low temperatures have a detrimental influence on larval swimming, which should be reflected in an increase in the value of energy expenditure $E_{S}$. Following the observations of Fuiman \& Batty (1997) of a linear decrease in swimming speed, a linear increase of energy expenditure with viscosity was assumed, and consequently $E_{S}$ was raised by $50 \%$ to $4.1 \times 10^{-6} \mathrm{cal} \mathrm{mg}^{-1} \mathrm{~mm}^{-1}$ at $\mathrm{v}=1.5 \times$ $10^{-6} \mathrm{~m}^{2} \mathrm{~s}^{-1}$ as compared to the value when $v=1.0 \times$ $10^{-6} \mathrm{~m}^{2} \mathrm{~s}^{-1}$. One would anticipate that $E_{S}$ should also rise as $\langle\varepsilon\rangle$ increases, as controlled swimming will become more difficult as the intensity of the turbulence increases, but quantitative measurements are lacking, so for the present no further change was imposed on $E_{S}$.

Fig. $4 \mathrm{a}$, b shows the results of the NEG calculations based on the predation rates discussed earlier for $\langle\varepsilon\rangle=$ $5.53 \times 10^{-9} \mathrm{~m}^{2} \mathrm{~s}^{-1}$ and $5.53 \times 10^{-8} \mathrm{~m}^{2} \mathrm{~s}^{-1}$, respectively. The first point to note in Fig. $4 \mathrm{a}$ is the replication of the result shown in Fig. 2 that a larva can gain most energy, not by swimming fast all of the time (which maximises its predation rate), but by adopting a pausetravel strategy akin to $1 / 2: 1 / 2$ (simulations) and

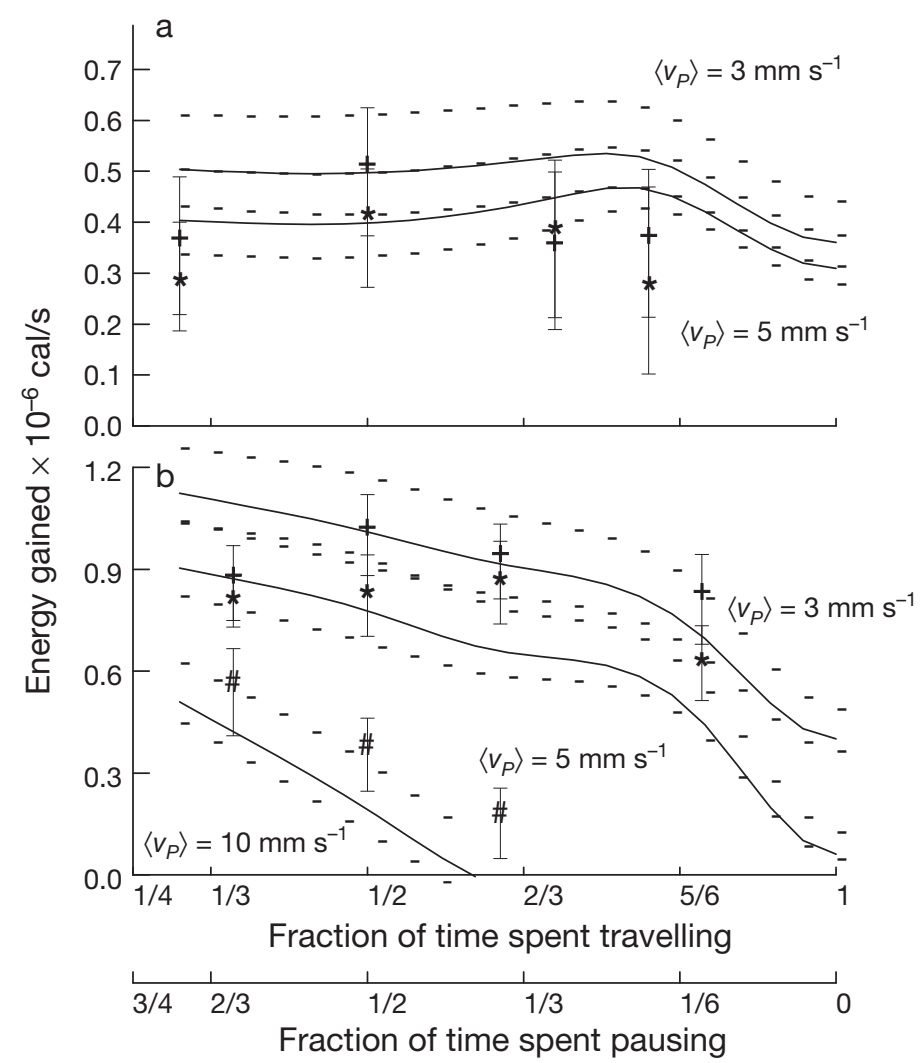

Fig. 4. Variation in the net energy gain against the pausetravel ratio and average travel speed based on the capture results shown in Fig. 3. (a) $\langle\varepsilon\rangle=5.53 \times 10^{-9} \mathrm{~m}^{2} \mathrm{~s}^{-3}$ and (b) $\langle\varepsilon\rangle=$ $5.53 \times 10^{-8} \mathrm{~m}^{2} \mathrm{~s}^{-3}$. Key as in Fig. 3. The swimming energy expenditure term has been raised by $50 \%$ to $E_{S}=4.1 \times$ $10^{-6} \mathrm{cal} \mathrm{mg}^{-1} \mathrm{~mm}^{-1}$, compared to the results shown in Fig. 2, to account for the increase in viscosity

between 1/3:2/3 and 1/6:5/6 (predictions), in line with the observations of Galbraith et al. (2004). Pause-travel foraging under this turbulence regime is robust with respect to changes in viscosity of the fluid. The second point to note is that the larva gains fractionally more energy when travelling at the lowest swimming speed of $3 \mathrm{~mm} \mathrm{~s}^{-1}$, although there is very little difference between these results and those at $5 \mathrm{~mm} \mathrm{~s}^{-1}$. The results when travelling at $10 \mathrm{~mm} \mathrm{~s}^{-1}$ are not shown here, simply because the calculations predict the associated NEG would be negative, i.e. the costs of swimming at such a speed outweigh the energy the larva can gain from the prey it can capture. The observations of Galbraith et al. (2004) suggest that average swimming speed in the travel mode is much closer to the values between 3 and $5 \mathrm{~mm} \mathrm{~s}^{-1}$, which are in line with the predictions for a viable pause-travel strategy. One is tempted to say that these results rule out the possibility of larva adopting a pause-travel strategy combined with such a relatively high swimming speed, but one must remember the uncertainty surrounding the 
estimate of $E_{S}$. A reduction of $50 \%$ in $E_{S}$ is sufficient to produce a positive NEG for a travel speed of $10 \mathrm{~mm} \mathrm{~s}^{-1}$, although this value remains significantly below those associated with the slower travel speeds.

The corresponding results for $\langle\varepsilon\rangle=5.53 \times 10^{-8} \mathrm{~m}^{2} \mathrm{~s}^{-3}$ are shown in Fig. 4b. This time the NEG for a $10 \mathrm{~mm} \mathrm{~s}^{-1}$ travel speed is positive (a consequence of the increased predation rate), but both the predictions and simulations indicate that any pause-travel strategy based on this speed is less efficient than simply cutting out the travel phase altogether and pausing all the time. Once again (with the caveats on the size of $E_{S}$ ) the conclusion must be that a pause-travel strategy associated with this speed is inefficient, and unlikely to be observed in practice. For the slower travel speeds, the simulations predict an optimum pause-travel strategy close to $1 / 2: 1 / 2$ (although the $2 / 3: 1 / 3$ and $1 / 3: 2 / 3$ give almost identical NEG values), again with the highest value of NEG associated with the $3 \mathrm{~mm} \mathrm{~s}^{-1}$ case. In a repetition of the results of Fig. $4 \mathrm{a}$, the pause-travel strategies associated with these travel speeds remain energetically robust, but this time when subjected to an increase in energy dissipation rate of the flow. This is an extremely important calculation. Larval fish are subject to ever changing environmental conditions, and lacking the capacity to make detailed calculations of the optimal response to these changes, any strategy they instinctively adopt must be effective across a range of turbulent regimes. These results show that pause-travelling at relatively slow travel speeds satisfies this criterion. The model predictions erroneously suggest that eliminating the travel phase altogether would be more beneficial, because the model significantly over-predicts the predation rate in the 2/3:1/3 mode (see Fig. 2). This is because the larval trajectories in this mode are most different from the idealised straight line trajectories on which the exact model calculations are based. It is extremely difficult to formulate a mechanistic model for $\left\langle t_{T R A}\right\rangle$, the average time a prey spends within the predator's narrow conical perception field, and hence to estimate the capture rate under these circumstances. The modelling ideas used in Section 3.2 of Lewis \& Bala (2006) to make these predictions could perhaps do with refinement in light of these results, but, overall, given all the complexities inherent in the problem, the results presented here are quite reasonable.

\section{Influence of energy dissipation rate and kinematic viscosity}

As pause-travel strategies are predicted by (Eq. 7) to be energetically viable only when associated with relatively low travel speeds, this discussion will be restricted to larval predators travelling at $3 \mathrm{~mm} \mathrm{~s}^{-1}$. Fig. 5 shows the

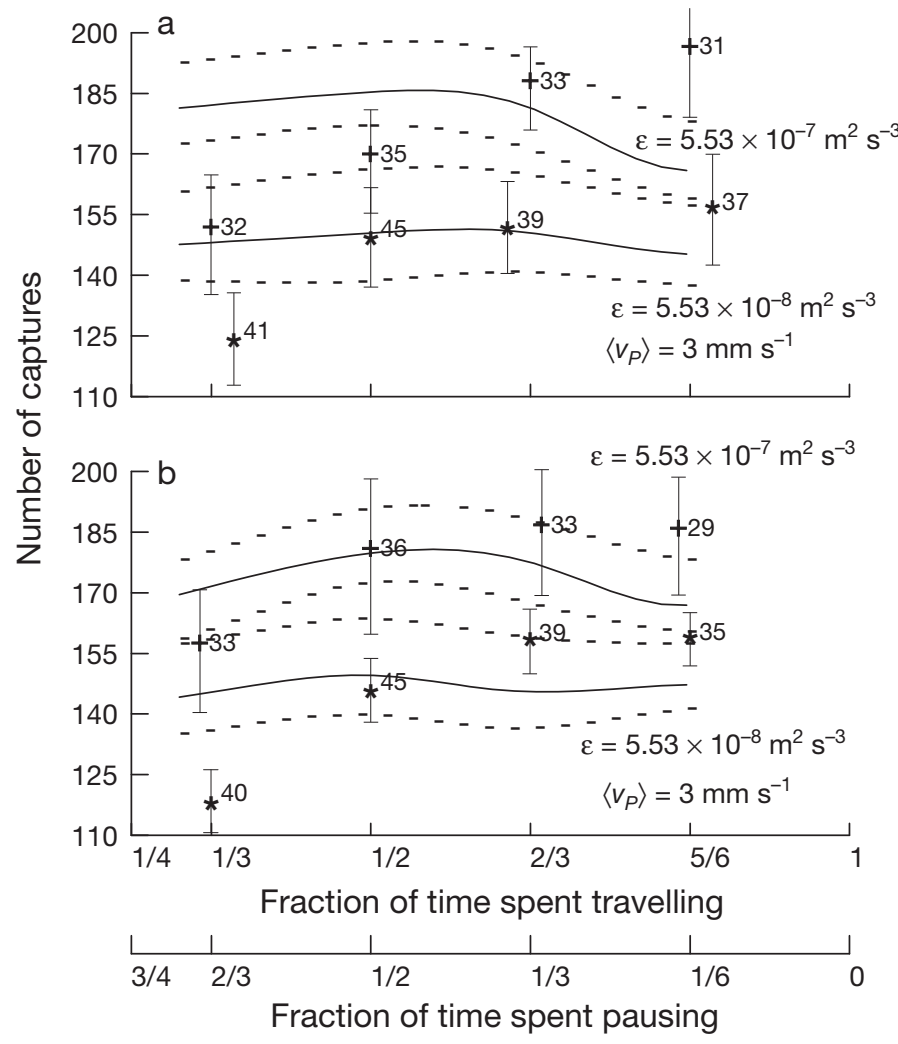

Fig. 5. Variation in the number of prey captures against the pause-travel ratio and energy dissipation rate made by 512 larvae swimming at an average travel speed of $3 \mathrm{~mm} \mathrm{~s}^{-1}$ in fluids of viscosities: (a) $v=1.5 \times 10^{-6} \mathrm{~m}^{2} \mathrm{~s}^{-1}$ and (b) $v=1.0 \times$ $10^{-6} \mathrm{~m}^{2} \mathrm{~s}^{-1}$. In each figure, an asterisk represents a simulation result for $\langle\varepsilon\rangle=5.53 \times 10^{-8} \mathrm{~m}^{2} \mathrm{~s}^{-3}$, whilst a plus sign represents that for $\langle\varepsilon\rangle=5.53 \times 10^{-7} \mathrm{~m}^{2} \mathrm{~s}^{-3}$. Otherwise, the key as in Fig. 3

number of captures made by 512 such predators foraging in turbulence regimes characterised by dissipation rates of $\langle\varepsilon\rangle=5.53 \times 10^{-8} \mathrm{~m}^{2} \mathrm{~s}^{-3}$ and $5.53 \times 10^{-7} \mathrm{~m}^{2} \mathrm{~s}^{-1}$, and viscosity values of $v=1.5 \times 10^{-6} \mathrm{~m}^{2} \mathrm{~s}^{-1}$ (Fig. $5 \mathrm{a}$ ) and $1.0 \times$ $10^{-6} \mathrm{~m}^{2} \mathrm{~s}^{-1}$ (Fig. 5b). (Some of the data points appear slightly offset because variations in the Kolmogorov time scales, as defined in Table 1, do not allow the corresponding non-dimensional pause-travel step equivalent to $3 \mathrm{~s}$ to be exactly divisible into sixths.) Changing the viscosity brings about an almost negligible difference in the number of captures the predators make. As in Fig. 3, significantly more captures are made foraging in the higher dissipation rate regime, although the difference is not as large as the increase predicted when $\langle\varepsilon\rangle$ is raised from $5.53 \times 10^{-9} \mathrm{~m}^{2} \mathrm{~s}^{-3}$ to $5.53 \times 10^{-8} \mathrm{~m}^{2} \mathrm{~s}^{-3}$ (roughly increasing by a factor of 1.6 in the former instance, but only by a factor 1.2 here).

Translating these predation rate predictions into estimates of NEG produces the results shown in Fig. 6a $\left(v=1.5 \times 10^{-6} \mathrm{~m}^{2} \mathrm{~s}^{-1}\right)$ and Fig. $6 \mathrm{~b}\left(v=1.0 \times 10^{-6} \mathrm{~m}^{2} \mathrm{~s}^{-1}\right)$. Once again, the simulations predict that following 


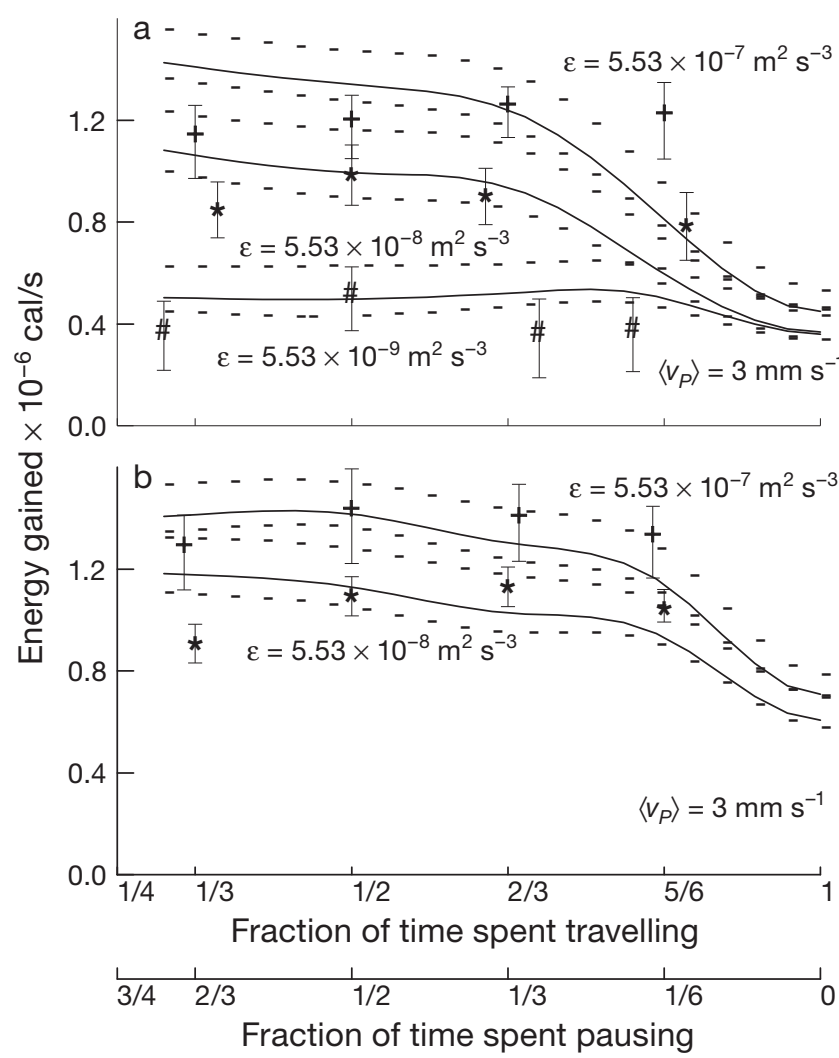

Fig. 6. Variation in the net energy gain against the pause-travel ratio and energy dissipation rate based on the capture results shown in Fig. 5: (a) $v=1.5 \times 10^{-6} \mathrm{~m}^{2} \mathrm{~s}^{-1}$ and (b) $\mathrm{v}=1.0 \times 10^{-6} \mathrm{~m}^{2}$ $\mathrm{s}^{-1}$. The swimming energy expenditure term is $E_{S}=4.1 \times 10^{-6} \mathrm{cal}$ $\mathrm{mg}^{-1} \mathrm{~mm}^{-1}$ in (a) and is $E_{S}=2.73 \times 10^{-6} \mathrm{cal} \mathrm{mg}^{-1} \mathrm{~mm}^{-1}$ in (b). In each figure, a hash mark represents a result for $\langle\varepsilon\rangle=5.53 \times 10^{-9}$ $\mathrm{m}^{2} \mathrm{~s}^{-3}$, an asterisk for $\langle\varepsilon\rangle=5.53 \times 10^{-8} \mathrm{~m}^{2} \mathrm{~s}^{-3}$ and a plus sign for $\langle\varepsilon\rangle=5.53 \times 10^{-7} \mathrm{~m}^{2} \mathrm{~s}^{-3}$. Otherwise, the key as for Fig. 3

strategies between $1 / 2: 1 / 2$ and $1 / 3: 2 / 3$ are (marginally) energetically most favourable in all cases. As the cost of swimming is assumed to be greater in the higher viscosity fluid, the NEG values in Fig. 6a are somewhat less than the corresponding values shown in Fig. 6b, even though the predation rates were very similar. Another point to note is that as a consequence of the rate of increase in predation rate with $\langle\varepsilon\rangle$ slowing down, as remarked upon in the last paragraph, the NEGs for $\langle\varepsilon\rangle=5.53 \times 10^{-7} \mathrm{~m}^{2} \mathrm{~s}^{-3}$ are only marginally above those predicted for $\langle\varepsilon\rangle=5.53 \times 10^{-8} \mathrm{~m}^{2} \mathrm{~s}^{-3}$. A much greater difference between the latter result and the prediction for $\langle\varepsilon\rangle=5.53 \times 10^{-9} \mathrm{~m}^{2} \mathrm{~s}^{-3}$ is apparent in Fig. 6a. This coming together of the NEG predictions for the 2 higher $\langle\varepsilon\rangle$ values has potentially important implications. To appreciate this, one must remember the assumption underlying the results of Fig. 6, namely that the swimming $\operatorname{cost} E_{S}$ is independent of $\langle\varepsilon\rangle$. In reality this cannot be correct, as it must cost a larva significantly more energy to perform controlled swimming motions, when subjected to increasing levels of turbulence. The small differential between the NEG when $\langle\varepsilon\rangle=5.53 \times 10^{-8} \mathrm{~m}^{2} \mathrm{~s}^{-3}$ and $\langle\varepsilon\rangle=5.53 \times 10^{-7} \mathrm{~m}^{2} \mathrm{~s}^{-3}$ implies that factoring in such a cost will cause the NEG in the latter regime to fall below that of the former, i.e. it will become disadvantageous for a larva to continue to pursue pause-travel foraging as the intensity of the turbulence increases.

Fig. $7 \mathrm{a}, \mathrm{b}$ illustrates this very point when the baseline values of $E_{S}$ are allowed to increase by a factor of $50 \%$ between the $\langle\varepsilon\rangle=5.53 \times 10^{-9} \mathrm{~m}^{2} \mathrm{~s}^{-3}$ and $\langle\varepsilon\rangle=5.53 \times$ $10^{-8} \mathrm{~m}^{2} \mathrm{~s}^{-3}$ regimes, and by a further $50 \%$ from $\langle\varepsilon\rangle=$ $5.53 \times 10^{-8} \mathrm{~m}^{2} \mathrm{~s}^{-3}$ to $\langle\varepsilon\rangle=5.53 \times 10^{-7} \mathrm{~m}^{2} \mathrm{~s}^{-3}$. (This loglinear increase in swimming cost with $\langle\varepsilon\rangle$ is purely speculative assumption, but probably not too unrea-

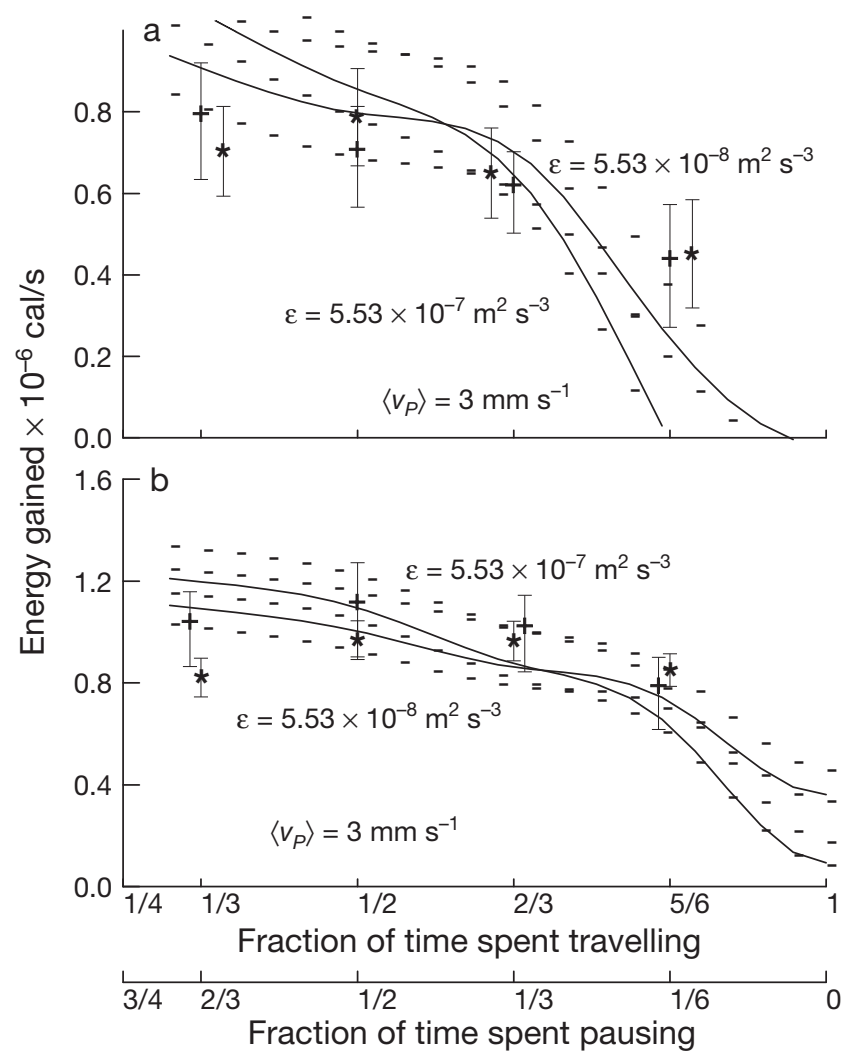

Fig. 7. Variation in the net energy gain against the pausetravel ratio and energy dissipation rate based on the capture results shown in Fig. 5. (a) The swimming energy expenditure terms are $E_{S}=6.14 \times 10^{-6} \mathrm{cal} \mathrm{mg}^{-1} \mathrm{~mm}^{-1}$ in the case when $\langle\varepsilon\rangle=$ $5.53 \times 10^{-8} \mathrm{~m}^{2} \mathrm{~s}^{-3}$ (simulation points marked by an asterisk; lower prediction curve when pause fraction is high), and $E_{S}=$ $9.21 \times 10^{-6} \mathrm{cal} \mathrm{mg}^{-1} \mathrm{~mm}^{-1}$ when $\langle\varepsilon\rangle=5.53 \times 10^{-7} \mathrm{~m}^{2} \mathrm{~s}^{-3}$ (simulation points marked by a plus sign; upper prediction curve when pause fraction is high). (b) The swimming energy expenditure terms are $E_{S}=4.1 \times 10^{-6} \mathrm{cal} \mathrm{mg}^{-1} \mathrm{~mm}^{-1}$ in the case when $\langle\varepsilon\rangle=5.53 \times 10^{-8} \mathrm{~m}^{2} \mathrm{~s}^{-3}$ (simulation points marked by an asterisk; lower prediction curve when pause fraction is high), and $E_{S}=6.14 \times 10^{-6} \mathrm{cal} \mathrm{mg}^{-1} \mathrm{~mm}^{-1}$ when $\langle\varepsilon\rangle=5.53 \times$ $10^{-7} \mathrm{~m}^{2} \mathrm{~s}^{-3}$ (simulation points marked by a plus sign; upper prediction curve when pause fraction is high) 
sonable; the precise definition of $E_{S}$ is not particularly conducive to a dimensional analysis approach to estimating a dependence on $\langle\varepsilon\rangle$.) The effect of these increases in $E_{S}$ is to bring together the NEG values for the 2 largest $\langle\varepsilon\rangle$ values so that they are overlapping. Indeed on Fig. $7 \mathrm{a}$ the $\langle\varepsilon\rangle=5.53 \times 10^{-8} \mathrm{~m}^{2} \mathrm{~s}^{-3}$ simulations (marked by an asterisk, as opposed to a plus sign for $\langle\varepsilon\rangle=5.53 \times 10^{-7} \mathrm{~m}^{2} \mathrm{~s}^{-3}$ ) now produce higher estimates of NEG in all but the 2/3:1/3 pause-travel mode. These results are replicated by the theoretical predictions, which have the $\langle\varepsilon\rangle=5.53 \times 10^{-7} \mathrm{~m}^{2} \mathrm{~s}^{-3} \mathrm{NEG}$ curve initially lying above the corresponding $\langle\varepsilon\rangle=5.53 \times$ $10^{-8} \mathrm{~m}^{2} \mathrm{~s}^{-3}$ curve when the proportion of pausing is large, but falling below as the travel proportion is increased. Similar results are shown in Fig. $7 \mathrm{~b}$ in the case when $v=1.0 \times 10^{-6} \mathrm{~m}^{2} \mathrm{~s}^{-1}$. Assuming this trend continues for higher values of $\langle\varepsilon\rangle$, one would expect to see continued reductions in NEG, leading to the conclusion that pause-travel foraging would become increasingly uneconomic for larvae in regions subject to high levels of turbulence.

Observational data of the influence of turbulence on larval fish foraging is relatively rare. Galbraith et al. (2004) highlight some experimental observations of Chamorro (2001) on the swimming behaviour of $10 \mathrm{~mm}$ fathead minnow larvae under turbulent conditions at $25^{\circ} \mathrm{C}$. These larvae are larger than the idealised cod larvae simulated here, and their swimming speeds were greater, up to about $40 \mathrm{~mm} \mathrm{~s}^{-1}$. Interestingly the average larval swimming speeds initially increased as the turbulent velocity increased from zero to about $10 \mathrm{~mm} \mathrm{~s}^{-1}$. (As a rough comparison, the Eulerian turbulent velocity scale $W$ for a flow field with $\langle\varepsilon\rangle=5.53 \times$ $10^{-7} \mathrm{~m}^{2} \mathrm{~s}^{-3}$ and $v=1.0 \times 10^{-6} \mathrm{~m}^{2} \mathrm{~s}^{-1}$ is about $7.4 \mathrm{~mm} \mathrm{~s}^{-1}$; see Table 2.) However, as the turbulent velocity was increased still further, to $25 \mathrm{~mm} \mathrm{~s}^{-1}$, the average larval swimming speed fell back to close to zero. This is perhaps indicative that at the higher turbulence levels pause-travel foraging is ceasing to become economic in NEG terms (as the swimming costs increase and prey capture efficiency declines), to which the larval response is to cease active swimming. It would be very interesting to see more observational studies to investigate this hypothesis further. A further point in support of this analysis is the size of the parameter $\chi_{U} \in$ $[0,1]$, which encapsulates the ratio of strength of the turbulent flow to the average larval travel speed (see Lewis \& Bala 2006, their Eq. 22 for a precise definition). For $\langle\varepsilon\rangle=5.53 \times 10^{-7} \mathrm{~m}^{2} \mathrm{~s}^{-3}$ and $\left\langle v_{P}\right\rangle=3 \mathrm{~mm} \mathrm{~s}^{-1}, \chi_{U} \approx 0.66$, indicating that the turbulent flow makes up about twothirds of the total velocity field determining the larva's trajectory via Eq. (4). At this kind of level an individual larva will start to lose the ability to direct and control its movements, or respond effectively to any potential prey entering its perception field. It will become more akin to a fluid particle randomly advected by the flow. Consequently, it seems highly unlikely that a cod larva of the size discussed will want to expend any extra energy trying to swim under such conditions. For larger larvae, which have the ability to travel at higher speeds, the point at which foraging ceases to become economic in terms of NEG, and when directional control becomes problematic, will occur at somewhat greater $\langle\varepsilon\rangle$ values. However, it seems unlikely that any fish larvae will be able to employ a pause-travel strategy effectively when subject to turbulence regimes characterised by energy dissipation rates much beyond $\langle\varepsilon\rangle \approx 10^{-6} \mathrm{~m}^{2} \mathrm{~s}^{-3}$

\section{CONCLUSIONS}

Saltatory or pause-travel foraging is employed by many different larval fish species, over a variety of temperature and flow regimes. Given this observation, and the limited capability of small fish larvae to perceive changes in locality and make sophisticated calculations of appropriate responses, one might expect that their adopted foraging strategy should remain fairly robust to changes in their fluid environment. This idea was tested by computing the predation rates of larval predators, following a variety of pause-travel strategies, in a number of different turbulent environments. The main conclusions are as follows:

(1) Saltatory foraging is intimately bound up with the relatively limited perception fields possessed by the fish larva. If fish larvae possessed spherical or even hemispherical fields of vision, as has often been assumed for simplicity in the past, pause-travel movements would not be observed. Larvae with such wide perception fields would be able to capture sufficient prey to more than support the energy expenditure required to spend all their foraging time in travel mode (see Fig. 2). The fact that pause-travel foraging is observed is an indication that their limited perception field does not allow them to encounter and capture enough prey to support the energy demands of constant travelling.

(2) Given that Point 1 is a necessary condition for saltatory foraging, the simulations were limited to larval fish predators with narrow conical perception fields. For the 2 slowest travel speeds, 3 and $5 \mathrm{~mm} \mathrm{~s}^{-1}$, pause-travel did indeed prove to be a robust strategy for these larvae to adopt. Detailed calculations from the simulations suggest that the 1/2:1/2 pause-travel mode is the most energetically favourable across the different viscosity and energy dissipation rate regimes, although the differences between this and the 2/3:1/3 and 1/3:2/3 modes are well within the uncertainty estimates. There is no well-defined optimal strategy, and hence it should not be too surprising that the obser- 
vations mentioned in the 'Introduction' record larval movements conforming to different foraging modes in this range. Swimming at $10 \mathrm{~mm} \mathrm{~s}^{-1}$ appears to be too energetically draining for the larvae to support any of the pause-travel modes (mindful of the uncertainties surrounding $E_{S}$ ). To support such a speed the larvae would need to find more energetically nutritious prey than Calanus finmarchius nauplii or significantly improve their capture efficiency by some energetically economic means. Given their physiological limitations, neither of these possibilities seems particularly probable.

(3) Increases in kinematic viscosity associated with lower temperatures reduce the overall NEG of the larvae. However, viscosity does not influence the fact that the marginal optimal foraging strategy lies closest to the 1/2:1/2 mode. Increases in energy dissipation rate, associated with stronger wind forcing, have a slight tendency to increase the proportion of pausing in the optimal mode. However, it is of more significance that the combined effect of the increasing energy demands required for swimming in such environments, coupled with declines in capture efficiency, will lead to reductions in NEG at high $\langle\varepsilon\rangle$ values. It is unlikely that the larvae can sustain economical pause-travel strategies under such circumstances. In all probability they will cease active swimming altogether (Chamorro 2001), or perhaps seek calmer parts of the water column, as copepods are observed to increase their escape responses when subjected to enhanced levels of turbulence (Tiselius \& Jonsson 1990, Fields \& Yen 1997, Viitasalo et al. 1998, Kiørboe et al. 1999).

One factor not discussed to any extent here is the influence of variations in the local prey density. The simulations conducted here were for a relatively low prey density of $4.3 \mathrm{l}^{-1}$. Uniform increases in prey density should not materially alter the conclusions presented here, as predation rate is directly proportional to prey density. Consequently, NEG levels would be raised for all travel speeds without materially altering the differentials between them, and slow swimming should still be favoured. Higher prey densities will tend to bias the pause-travel modes towards an increased proportion of travelling, although calculations carried out at a prey density of $100 \mathrm{l}^{-1}$ by Lewis \& Bala (2006) suggest that this effect is very marginal.

Pitchford et al. (2003) put forward the hypothesis that larvae may take advantage of patchiness in their prey distribution, i.e. they should swim faster when they enter a volume abundant in prey, because the potential energy gains they can make will sustain this higher speed. This presupposes that the larvae, with their limited perception fields, are capable of 'surveying' a sufficient volume of the water column to discern that certain regions contain significantly more prey than others and adjust their foraging strategies accordingly. To the present authors this seems an unlikely scenario, particularly as the mixing properties of turbulence will tend to smooth out a heterogeneous prey distribution in any case. It would seem more likely that fish larvae perform pause-travel foraging because this is a robust, effective strategy when prey density is marginal for survival, and any changes in larval swimming behaviour on entering a region abundant with prey will be brought about by satiation (e.g. the decline in swimming activity observed by Munk 1995 as the number of nauplii in the gut increased). However, more experimental observations of fish larvae in turbulent environments are necessary before definitive conclusions can be made regarding these points.

Acknowledgements. S. I. Bala acknowledges the financial support of Bayero University, Kano, Nigeria.

\section{LITERATURE CITED}

Batchelor GK (1953) The theory of homogeneous turbulence. Cambridge University Press, Cambridge

- Browman HI, O'Brien WJ (1992a) The ontogeny of search behaviour in the white crappie, Pomoxis annularis. Environ Biol Fishes 34:181-195

Browman HI, O'Brien WJ (1992b) Foraging and prey search behaviour of golden shiner (Notemigonus crysoleucas) larvae. Can J Fish Aquat Sci 49:813-819

Browman HI, Skiftesvik AB (1996) Effects of turbulence on the predation cycle of fish larvae: comments on some of the issues. Mar Ecol Prog Ser 139:309-312

Bundy MH, Gross TF, Vanderploeg HA, Strickler JR (1998) Perception of inert particles by calanoid copepods: behavioural observations and a numerical model. J Plankton Res 20:2129-2152

Chamorro VC (2001) The effects of small scale turbulence in the feeding ecology and swimming speed of fathead minnow larvae (Pimephales promelas), inland silverside larvae (Menidia beryllina) and the lobate ctenophore (Mnemiopsis leidyi). Master's thesis, University of Maryland, College Park, MD

Coughlin DJ, Strickler JR, Sanderson B (1992) Swimming and search behaviour in clownfish, Amphiprion perideraion larvae. Anim Behav 44:427-440

Fields DM, Yen J (1997) Implications of the feeding current structure of Eucheata rimana, a carnivorous copepod, on the spatial orientation of their prey. J Plankton Res 19:79-95

Fiksen Ø, Utne ACW, Aksnes DL, Eiane K, Helvik JV, Sundby S (1998) Modelling the influence of light, turbulence and development on foraging in larval cod and herring. Fish Oceanogr 7:355-363

Fuiman LA, Batty RS (1997) What a drag it is getting cold: partitioning the physical and physiological effects of temperature on fish swimming. J Exp Biol 200:1745-1755

Fung JCH, Hunt JCR, Malik NA, Perkins RJ (1992) Kinematic simulations of homogeneous turbulence by unsteady random Fourier modes. J Fluid Mech 236:281-318

Galbraith PS, Browman HI, Racca RG, Skiftesvik AB, SaintPierre JF (2004) The effect of turbulence on the energetics of foraging in Atlantic cod (Gadus morhua) larvae. Mar Ecol Prog Ser 281:241-257

Gerritsen J, Strickler JR (1977) Encounter probabilities and 
community structure in zooplankton: a mathematical model. J Fish Res Board Can 34:73-82

Kiørboe T, Saiz E, Visser AW (1999) Hydromechanical signal perception in the copepod Acartia tonsa. Mar Ecol Prog Ser 179:97-111

Leising AW, Franks PJS (1999) Larval Atlantic cod (Gadus morhua) and haddock (Melanogrammus aeglefinus) growth on Georges Bank: a model with temperature, prey size and turbulence forcing. Can J Fish Aquat Sci 56: 25-36

Lewis DM (2003) Planktonic encounter rates in homogeneous isotropic turbulence: the case of predators with limited fields of sensory perception. J Theor Biol 222:73-97

> Lewis DM, Bala SI (2006) Plankton predation rates in turbulence: a study of the limitations imposed on a predator with a non-spherical field of sensory perception. J Theor Biol 242:44-61

Lewis DM, Pedley TJ (2000) Planktonic contact rates in homogeneous isotropic turbulence: theoretical predictions and kinematic simulations. J Theor Biol 205:377-408

Lewis DM, Pedley TJ (2001) The influence of turbulence on plankton predation strategies. J Theor Biol 210:347-365

Lough RG, Buckley LJ, Werner FE, Quinlan JA, Pehrson Edwards K (2005) A general biophysical model of larval cod growth: comparison of May 1993 vs. 1994 observations on Georges Bank. Fish Oceanogr 14:241-262

MacKenzie BR, Kiørboe T (1995) Encounter rates and swimming behaviour of pause-travel and cruise larval fish predators in calm and turbulent laboratory environments. Limnol Oceanogr 40:1278-1289

MacKenzie BR, Kiørboe T (2000) Larval fish feeding and turbulence: a case for the downside. Limnol Oceanogr 45: $1-10$

MacKenzie BR, Leggett WC (1991) Quantifying the contribution of small-scale turbulence to the encounter rates between larval fish and their zooplankton prey: effects of wind and tide. Mar Ecol Prog Ser 73:149-160

MacKenzie BR, Miller TJ, Cyr S, Leggett WC (1994) Evidence for a dome shaped relationship between turbulence and larval fish ingestion rates. Limnol Oceanogr 39:1790-1799

Malik NA, Vassilicos JC (1999) A Lagrangian model for turbulent dispersion with turbulent like flow structure: comparison with direct numerical simulation for two-particle statistics. Phys Fluids 11:1572-1580

Mann J, Ott S, Pécseli, HL, Trulsen J (2002) Predator-prey encounters in turbulent waters. Phys Rev E 65:026304

Mann J, Ott S, Pécseli HL, Trulsen J (2006) Laboratory studies related to predator-prey encounters in turbulent environments: effects of changes in orientations and fields of view. J Plankton Res 28:509-522

Mei R, Adrian RJ (1995) Effect of Reynolds number on isotropic turbulent dispersion. Trans ASME I: J Fluids Eng 117:402-409

Metcalfe AM, Pedley TJ, Thingstad TF (2004) Incorporating turbulence into a plankton foodweb model. J Mar Syst 49: 105-122

Muelbert JH, Lewis MR, Kelley DE (1994) The importance of small-scale turbulence in the feeding of herring larvae. J Plankton Res 16:927-944

Munk P (1995) Foraging behaviour of larval cod (Gadus morhua) influenced by prey density and hunger. Mar Biol

Editorial responsibility: Roger Hughes,

Bangor, UK
122:205-212

Paul AJ (1983) Light, temperature, nauplii concentrations and prey capture by first feeding pollock larvae Theragra chalograma. Mar Ecol Prog Ser 13:175-179

> Peck MA, Buckley LJ, Bengtson DA (2006) Effects of temperature on the swimming speed of larval and juvenile Atlantic cod (Gadus morhua): implications for individualbased modelling. Environ Biol Fishes 75:419-429

Pitchford JW, James A, Brindley J (2003) Optimal foraging in patchy turbulent environments. Mar Ecol Prog Ser 256: 99-110

Porter SM, Ciannelli L, Hillgruber N, Bailey KM, Kung-Sik Chang, Canino M, Haldorson LJ (2005) Environmental factors influencing larval walleye pollock Theragra chalcogramma feeding in Alaskan waters. Mar Ecol Prog Ser 302:207-217

Rosenthal H, Hempel G (1970) Experimental studies in feeding and food requirements of herring larvae (Clupea harengus). In: Stelle JH (ed) Marine food chains. Oliver and Boyd, Edinburgh

Rothschild BJ, Osborn TR (1988) Small-scale turbulence and plankton contact rates. J Plankton Res 10:465-474

Sundby S, Fossum P (1990) Feeding conditions of Arcto-norwegian cod larvae compared with the Rothschild-Osborn theory on small scale turbulence and planktonic contact rates. J Plankton Res 12:1153-1162

Tennekes H, Lumley JL (1972) A first course in turbulence. MIT Press, Cambridge, MA

> Tiselius P, Jonsson P (1990) Foraging behaviour of six calanoid copepods: observations and hydrodynamic analysis. Mar Ecol Prog Ser 66:23-33

> Viitasalo M, Kiørboe T, Flinkman J, Pedersen LW, Visser AW (1998) Predation vulnerability of planktonic copepods: consequences of predator foraging strategies and prey sensory abilities. Mar Ecol Prog Ser 175:129-142

> Visser AW, Jackson GA (2004) Characteristics of the chemical plume behind a sinking particle in a turbulent water column. Mar Ecol Prog Ser 283:55-71

- Visser AW, MacKenzie BR (1998) Turbulence-induced contact rates of plankton: the question of scale. Mar Ecol Prog Ser 166:307-310

von Herbing IH (2002) Effects of temperature on larval fish swimming performance: the importance of physics to physiology. J Fish Biol 61:865-876

von Herbing IH, Boutilier RG (1996) Activity and metabolism of larval Atlantic cod (Gadus morhua) from Scotian Shelf and Newfoundland source populations. Mar Biol 124:607-617

von Herbing IH, Gallager SM (2000) Foraging behaviour in early Atlantic cod larvae (Gadus morhua) feeding on a protozoan (Balanion sp.) and a copepod nauplius (Pseudodiaptomus sp.). Mar Biol 136:591-602

von Herbing IH, Gallager SM, Halteman W (2001) Metabolic costs of pursuit and attack in early larval Atlantic cod. Mar Ecol Prog Ser 216:201-212

Wieser W, Kaufmann R (1998) A note on interactions between temperature, viscosity, body size and swimming energetics in fish larvae. J Exp Biol 201:1369-1372

> Yamazaki H, Osborn TR, Squires KD (1991) Direct numerical simulation of planktonic contact rate in turbulent flow. J Plankton Res 13:629-643

Submitted: August 6, 2007; Accepted: November 14, 2007 Proofs received from author(s): April 17, 2008 